\title{
Electronic structure of reconstructed Au(100): Two-dimensional and one-dimensional surface states
}

\author{
S. Bengió, ${ }^{1,{ }^{*}}$ V. Navarro, ${ }^{2}$ M. A. González-Barrio, ${ }^{2,3}$ R. Cortés,${ }^{2}$ I. Vobornik, ${ }^{4}$ E. G. Michel, ${ }^{1}$ and A. Mascaraque ${ }^{2,3}$ \\ ${ }^{1}$ Departamento de Física de la Materia Condensada, Universidad Autónoma de Madrid, 28049 Madrid, Spain \\ ${ }^{2}$ Departamento de Física de Materiales, Universidad Complutense de Madrid, 28040 Madrid, Spain \\ ${ }^{3}$ Unidad Asociada IQFR(CSIC)-UCM, 28040 Madrid, Spain \\ ${ }^{4}$ Istituto Officina dei Materiali (IOM)-CNR, Laboratorio TASC, in Area Science Park, S.S.14, Km 163.5, I-34149 Trieste, Italy
}

(Received 4 May 2012; revised manuscript received 28 June 2012; published 18 July 2012)

\begin{abstract}
The clean surface of $\mathrm{Au}(100)$ presents a complex reconstruction characterized by a hexagonal topmost layer. We report an angle-resolved photoemission study of the electronic structure of this surface, including an analysis of the Fermi surface, combined with structural information from low-energy electron diffraction and scanning tunneling microscopy. In the complex Fermi surface map found, we identify different contributions from the bulk bands, from interface states located below the hexagonal topmost layer, and from the hexagonal topmost layer itself. The electronic states related to this layer exhibit quasi-one-dimensional character, in agreement with the chain aspect of the reconstructed layer, as demonstrated by their dispersion, periodicity, and reciprocal space location.
\end{abstract}

DOI: 10.1103/PhysRevB.86.045426

PACS number(s): 68.35.Ja, 71.15.Ap, 68.35.Rh, 71.15.Nc

\section{INTRODUCTION}

The most evident similarity between the clean $\mathrm{Au}, \mathrm{Pt}$, and $\operatorname{Ir}(100)$ reconstructed surfaces is the hexagonal symmetry of their topmost layer. ${ }^{1}$ This is an intriguing property that has received ample attention, and different techniques have been applied in order to understand the structural properties of these surfaces, and in particular of the $\mathrm{Au}(100)$ reconstruction. ${ }^{1-7}$ The mismatch between the topmost hexagonal layer and the substrate generates a strain that is released by lateral contraction and buckling of the hexagonal layer. The topmost hexagonal layer is highly modulated in the [1110] direction, with a fivefold periodicity. In the [110] direction, there is a smooth modulation that leads to fringes with a chainlike structure and a much longer periodicity.

High-symmetry faces of noble metals present Shockley surface states,${ }^{8-10}$ related to the bulk $s p$ band gap near the $L$ point. An interesting question is how the Shockley surface state expected for the $\mathrm{Au}(100)$ bulk-terminated surface is affected by the reconstruction. In the case of $\mathrm{Au}(111)$, evidence was found that the reconstruction acts as a superlattice on the Shockley surface state, modulating its electronic density, ${ }^{11}$ an indication that it is sensitive to surface modifications, such as reconstructions. High-resolution photoemission studies have found that it survives the reconstruction of the surface ${ }^{10}$ and that it replicates with its periodicity. ${ }^{12}$ This behavior leads to the formation of small gaps at the intersections of the original surface-state parabola and its replicas. In the case of $\mathrm{Au}(110)$, the $(2 \times 1)$ "missing row" reconstruction removes the analogous Shockley surface state by pushing it above the Fermi level. ${ }^{13}$ Early work on $\mathrm{Au}(100)$ found a deeper surface band related to the reconstruction. ${ }^{14}$ A structural analysis shows quasi-two-dimensional (2D) dislocations and strongly anisotropic diffusion of vacancies ${ }^{15}$ on this surface, which proves a relative decoupling of the hexagonal overlayer from the underlying, square-symmetry bulk and lateral confinement in the fringes. It is an open question as to whether this behavior is extended to the electronic states.

On the other hand, the ability of gold to form chains has been observed in many experiments. ${ }^{16,17}$ Numerical simulations for atomic-size metallic nanowires confirmed the stability of these structures for $\mathrm{Au}$, but did not find them in Ag. ${ }^{18}$ Takeuchi et al. ${ }^{19}$ have suggested that the role of $5 d$ electrons is crucial for the formation of the $\mathrm{Au}$ reconstruction, contributing to the bonding and cohesion through hybridization with the $s p$ band. As the reason for the formation of a $2 \mathrm{D}$ system in the reconstructed $\mathrm{Au}$ are the relativistic effects of the $s p$ electrons, ${ }^{19}$ it could also be the reason that explains the cohesion in a one-dimensional (1D) system since the consequences of these relativistic modifications in the metallic bonding would be even more pronounced for the one-dimensional chain structures. ${ }^{16}$ Again, how these features are reflected in the electronic structure of the reconstructed surface is unknown.

In this paper, we set out to study the electronic structure of the clean reconstructed $\mathrm{Au}(100)$ surface and to determine how its complex structural properties affect the electronic states and their periodicity, and in particular how the topmost hexagonal layer and the bulk-terminated $\mathrm{Au}(100)$ surface contribute to the electronic structure.

\section{EXPERIMENT}

The experiments were performed in ultrahigh-vacuum (UHV) chambers with a base pressure in the low $10^{-10} \mathrm{mbar}$ range. The $\mathrm{Au}(100)$ sample was cleaned by repeated 600 $1000 \mathrm{eV}$ Ar ions sputtering and annealing cycles $\left(\sim 600^{\circ} \mathrm{C}\right)$ until a sharp low-energy electron diffraction (LEED) pattern was observed. Atomic resolution images were taken with a homemade scanning tunneling microscope (STM) operated with a commercial RHK STM-100 control electronics.

Angle-resolved photoemission spectroscopy (ARPES) experiments were performed in an UHV chamber located at the low-energy branch of the APE-IOM beamline, ${ }^{20}$ equipped with a Scienta SES-2002 electron energy analyzer, and receiving synchrotron light from the Elettra storage ring (Trieste, Italy). The energy resolution was set to $60 \mathrm{meV}$ and the angle resolution was $0.12^{\circ}$. The quality of the surface was checked by measuring the surface state at the $\overline{\mathrm{X}}$ point. Symmetry points are referred to the surface Brillouin zone (SBZ) of unreconstructed $\mathrm{Au}(100)$. Fermi surface mapping 
was performed at constant photon energy with a two-degreesof-freedom manipulator, i.e., theta and phi angles could be manipulated in an independent way. The surface and bulk bands were mapped in a window of approximately $0.6 \mathrm{eV}$ below the Fermi energy. The polarization of the light was horizontal and coincided with the measuring plane. A $k$-space portion near the $\overline{\mathrm{M}}$ point was mapped with higher resolution in order to probe the electronic states close to the Fermi energy.

\section{RESULTS}

\section{A. Surface structure}

The topmost layer of reconstructed $\mathrm{Au}(100)$ is of quasihexagonal symmetry, as described above. Although the structural distortion affects several layers underneath, it is much larger for the topmost hexagonal layer. ${ }^{4,5}$ The topmost hexagonal layer is highly modulated in the [110] direction, with a fivefold periodicity. In the [110] direction, there is a smooth modulation that leads to fringes with a chainlike structure, and a periodicity that varies with the material. In the cases of $\mathrm{Au}(100)$ and $\mathrm{Pt}(100)$ the reconstruction is usually called $(5 \times 20)$ or $(5 \times 25)$, whereas $\operatorname{Ir}(100)$ exhibits a $(5 \times 1)$ reconstruction. By accurate measurement of the Bragg positions of the diffraction beams, Liew et al. obtained a $(5 \times m)$ superlattice in the case of $\mathrm{Au}(100)$, where $m$ ranges from 26 to $28 .{ }^{6}$ Previous transmission electron microscopy results $^{7}$ gave a broader range of values (from 20 to 36), centered around 28. The hexagonal layer has been described as rotated by a small angle $\left(\sim 0.7^{\circ}\right)$, so that the coincidence lattice would be only approximate and the layer would be incommensurate. ${ }^{4,5,7}$ In the case of $\operatorname{Pt}(100)$, both a rotated and a nonrotated reconstruction have been identified. We refer the reader to Ref. 21 for a recent overview of the energetics of $\mathrm{Au}(100)$ and $\mathrm{Pt}(100)$ reconstructions.

A STM image of the clean surface is shown in Fig. 1(a). The characteristic reconstruction fringes, $14.4 \AA$ wide and running along compact $\langle 110\rangle$ directions, are clearly seen. Six-atom rows of the top layer fit onto five-atom rows of the underlying substrate, as sketched in Fig. 1(b). The LEED pattern of Fig. 1(d) shows not only the fivefold periodicity along [110], but also the periodicity of the [110] modulation is reflected in the splitting of the $1 / 5,2 / 5,3 / 5$, and $4 / 5$ spots. Depending on the relative orientation of the hexagonal and square unit cells, reconstruction domains are possible. The fact that spots in one direction are more intense than in the perpendicular direction [Fig. 1(d)] reveals that the relative abundance of reconstruction domains is not the same. Although it is not shown in the image, reconstruction fringes run parallel to steps ${ }^{15}$ and terrace widths accommodate an integer number of reconstruction fringes. Both facts raise the question of the apparent close relationship between reconstruction, steps direction, and terrace width.

\section{B. Bulk-related electronic states}

Contributions from the bulk $s p$ of the substrate were identified in the Fermi surface map (FSM) of the reconstructed $\mathrm{Au}(100)$ at a photon energy $\mathrm{h} v=41 \mathrm{eV}$ [see Fig. 2(a)]. This is the only accessible bulk band at the Fermi energy. In all figures, photoemission intensity is represented in a reversed gray scale, i.e., black corresponds to high photoelectron intensity. In
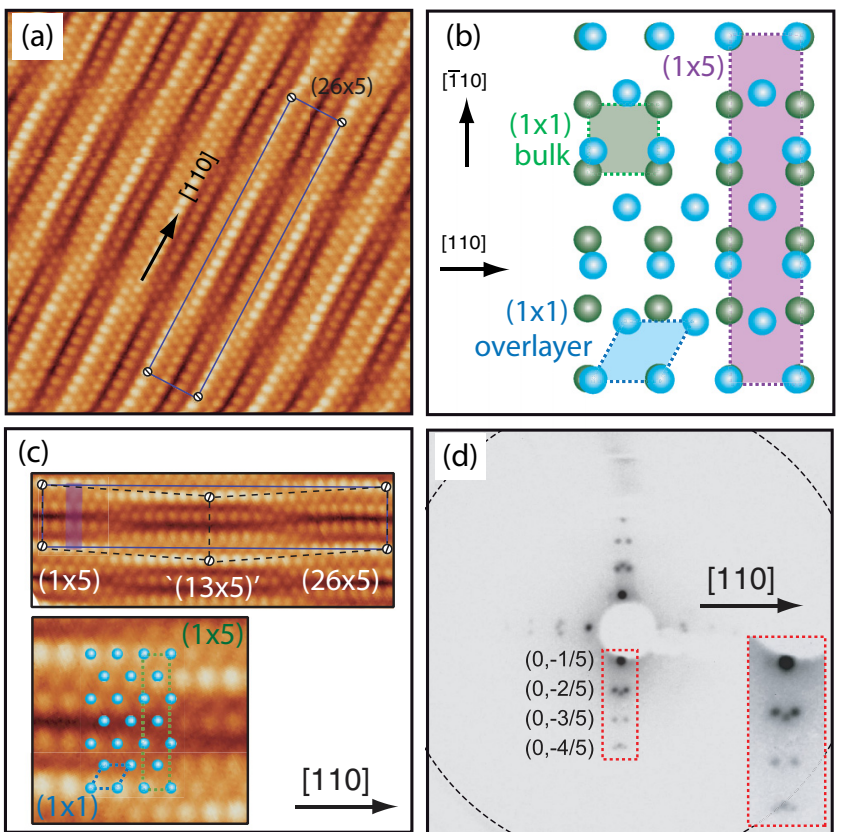

FIG. 1. (Color online) (a) $10 \mathrm{~nm} \times 10 \mathrm{~nm}$ STM image of the reconstructed $\mathrm{Au}(100)$ surface, showing the fringe structure. A conventional $(26 \times 5)$ unit cell is drawn in solid lines. (b) Top view of a schematic representation of the hexagonal array of atoms of the overlayer (blue) on the square array of the bulk-terminated $\mathrm{Au}(100)$ surface (green). A $(5 \times 1)$ unit cell is drawn for reference in purple dashed lines. (c) Detail of the reconstructed overlayer, showing the conventional $(26 \times 5)$ unit cell (continuous purple line) and $(13 \times 5)$ cells (black dashed line). The fringes direction in (a) and (c) is [110] for the considered domain orientation. (d) LEED pattern of the $\mathrm{Au}(100)$ surface taken at $30 \mathrm{eV}$, showing the two, perpendicular reconstruction domains, one of them predominant. The spots contained in the dashed rectangle are enlarged in the lower side edge. Note the different splitting for each spot.

Fig. 2(b), the theoretical $s p$ band $^{22}$ is plotted in green solid lines on the measured FSM. Electrons coming from the bulk $s p$ band undergo Umklapp processes in their way out of the solid, and interchange momentum with the topmost hexagonal lattice, giving rise to Umklapp replicas of the $s p$ bands, which are plotted in green dashed lines. The replicas highlighted are drawn by shifting the $s p$ bands in $k_{\|}$along [1힐 by $n \times$ $2 \pi / 5 a=n \times 0.44 \AA^{-1}, n$ being an integer, and $a=2.88 \AA$ the substrate surface square-cell size. The original $s p$ states and their replicas are mainly visible in certain regions of the FSM, notably near $k_{x}^{\|}=1.5-2.0 \AA^{-1}$. Several effects contribute to this enhanced intensity. First, in some regions of the FS, the $s p$ intensity is enhanced by nondirect transitions ${ }^{23,24}$ or by matrix-element effects and the same happens to the replicas. Second, the intensity of the replicas depends on the relative abundance of the reconstruction domains.

\section{2D surface electronic states}

Also clearly identifiable in the FSM is the Shockley surface state expected for the bulk-terminated substrate, recognizable by a characteristic oval at $\overline{\mathrm{X}}$, that results from cutting the binding energy (BE) versus $k_{\|}$paraboloid with a constant 


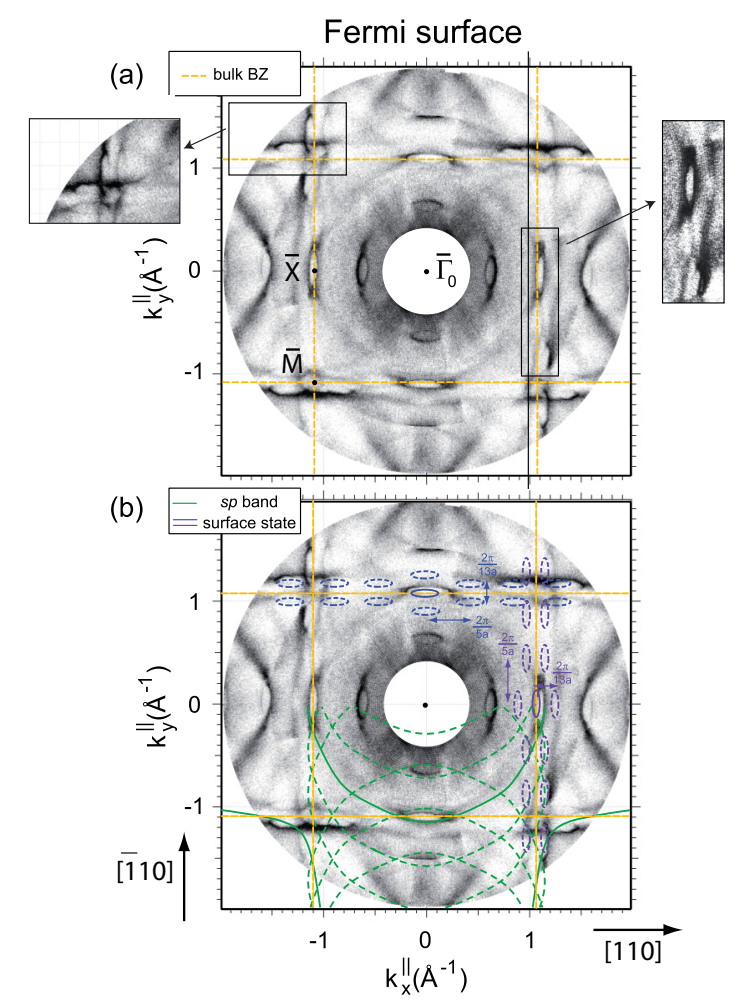

(c)

Band dispersion

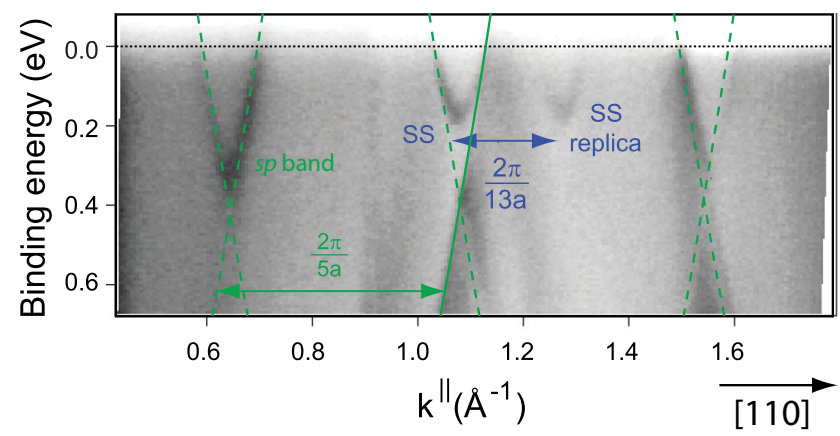

FIG. 2. (Color online) (a) Fermi surface map of the reconstructed $\mathrm{Au}(100)$ measured at $\mathrm{h} v=41 \mathrm{eV}$. Experimental data are measured for $180^{\circ}$ and symmetrized. Yellow (gray) lines correspond to the surface Brillouin zone edges. Two portions of the Fermi surface are enlarged and shown with enhanced contrast. (b) Theoretical $s p$ band and Shockley surface state are superimposed in green (large circles) and blue/violet (small ovals) solid lines, respectively. Replicas of these states are drawn in dashed lines (not all seen for the photon energy used in this Fermi surface map). Note that the feature detected at $k_{x}^{\|} \simeq 0.6 \AA^{-1}$ is not a replica but due to the cross of the Fermi level by a $s p$ band replica [see (c)]. (c) BE vs $k_{\|}$photoemission spectra along [110] with the theoretical $s p$ band in green solid line and its replicas in dashed lines at $\pm 2 \pi / 5 a= \pm 0.44 \AA^{-1}$. The Shockley surface state is seen at $\bar{X}$, as well as its replica at $+2 \pi / 13 a$.

energy plane. The substrate surface state is also affected by the topmost hexagonal layer potential and becomes likewise replicated due to Umklapp processes. Figure 2(c) shows a BE versus $k_{\|}$reversed gray scale representation along the [110] direction. As a reference, the theoretical $s p$ bands and their replicas are superimposed in green. We have plotted in dashed lines the replicated $s p$ states by the same procedure mentioned before, i.e., by shifting the original state in $k_{\|}$along [110] by $n \times 2 \pi / 5 a=n \times 0.44 \AA^{-1}$.

The surface state and its replicas are also drawn in Fig. 2(b). By shifting the surface state in $k_{\|}$along [110] and [110] by $2 \pi / 5 a=0.44 \AA^{-1}$ and $2 \pi / 13 a=0.168 \AA^{-1}$, respectively, the electronic features of the FSM highlighted in blue can readily be reproduced. Also, along [110] $\{[\overline{1} 10]$ for the perpendicular domain $\}$ the surface state was found to have 13-fold periodicity. For example, in Fig. 2(c) the Shockley surface state around the $\overline{\mathrm{X}}$ point $\left(k_{\|}=1.09 \AA^{-1}\right)$ and its replica at $+2 \pi / 13 a$ can be easily identified. A closer look at the STM image of Fig. 1(c) reveals that the complex surface unit cell, described with a conventional 26-fold periodicity, indeed is close to a 13-fold periodic lattice, highlighted with blue circles. Therefore, we can infer that the surface potential affecting the outgoing electrons contains a stronger 13-fold periodicity (seen in the Shockley surface state replica) and a smaller 26-fold periodicity, related to the registry difference of the corrugation between adjacent atomic lines along the [110] direction.

It is worth to remark that the substrate Shockley surface state persists after reconstruction and becomes an "interface state." We consider in the following the nature of the Shockley surface state replicas in the $\mathrm{Au}(100)$ face. In the $\mathrm{Au}(111)$ face, the reconstruction only involves a change in stacking of half unit cell, and the surface state persists and replicates as an initial-state superlattice effect. In the $\mathrm{Au}(100)$ face, the Shockley surface state also survives the reconstruction, but to some extent it is electronically decoupled from the topmost hexagonal layer due to their different symmetry. Further studies should be made to elucidate whether the replicas of the Shockley surface state seen are an initial- or final-state superlattice effect, but the experimental behavior observed by us is similar to the behavior of the bulk $s p$ bands, and thus there are no evidences supporting an initial-state origin for the replicas. There is still an all important issue to be considered, namely, whether there is any electronic state coming from the hexagonal topmost layer itself.

\section{1D electronic states}

In Fig. 3, constant energy maps are shown for a portion of reciprocal space covering two $\bar{M}$ points. At the Fermi level, the replicas of the bulk $s p$ band and of the Shockley surface state, coming from the two perpendicular domains, can be distinguished and dominate the map. Two arrows point towards the replicas of the Shockley surface state contours in the $-0.046-\mathrm{eV}$ panel. With increasing BE, the characteristic Shockley surface state oval shrinks and eventually disappears [for $0.159 \mathrm{eV} \mathrm{BE}$, consistent with the bottom of the Shockley surface state band as seen in Fig. 2(c)].

For higher BE, along with intensity coming from the $s p$ bulk band, there remain some linear, nondispersive (in $k_{\|}$, for constant energy) features in the neighborhood of the absolute $\overline{\mathrm{M}}$-gap area, which appear as horizontal and vertical lines (see panel for 0.364-eV BE). These linear features can not be explained in terms of either the bulk $s p$ states and their replicas (these states do disperse with $k_{\|}$and have a wholly different shape, see also below) or the Shockley surface state (no electrons coming from this electronic state appear at 


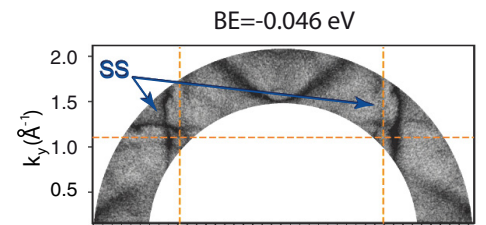

$\mathrm{BE}=0.082 \mathrm{eV}$

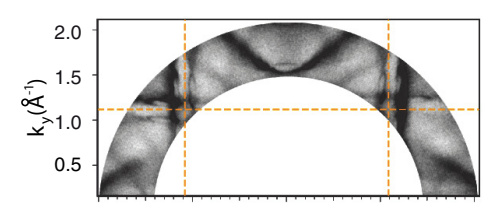

$\mathrm{BE}=0.287 \mathrm{eV}$
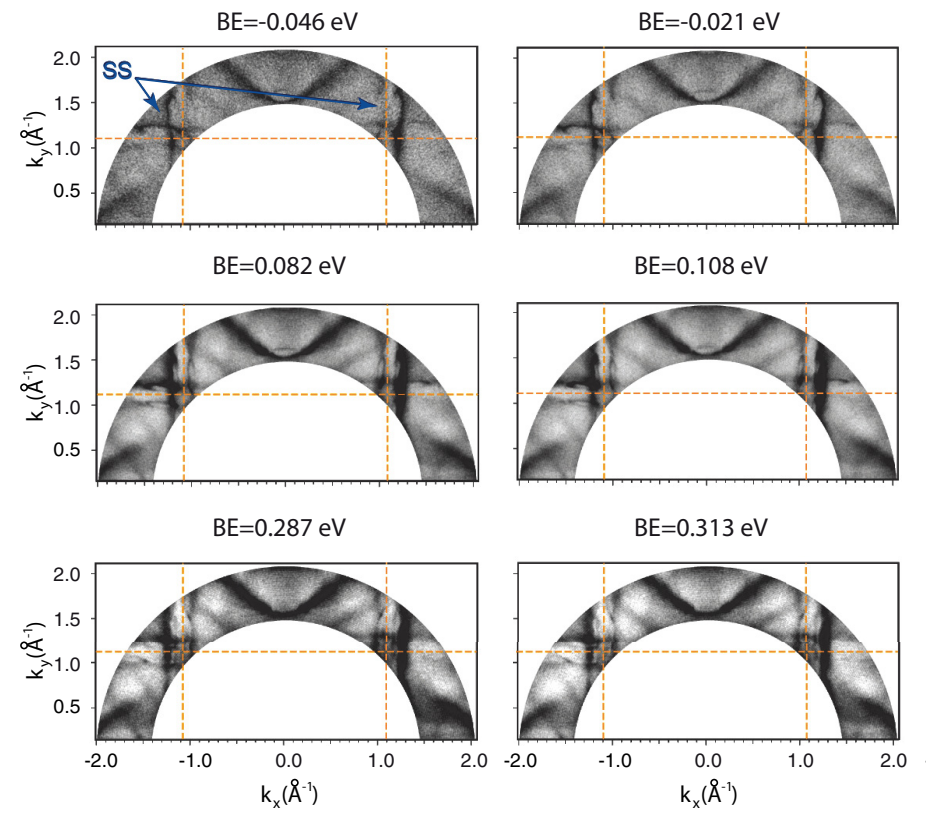

$\mathrm{BE}=0.108 \mathrm{eV}$

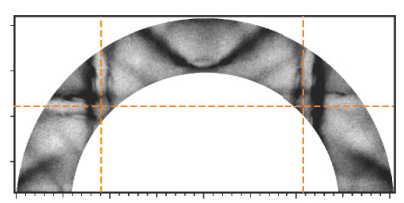

$\mathrm{BE}=0.313 \mathrm{eV}$
FIG. 3. (Color online) Constant energy maps measured with $\mathrm{h} v=41 \mathrm{eV}$ in areas covering two $\overline{\mathrm{M}}$ points. BEs run from $-0.046 \mathrm{eV}$ (very close to the Fermi level) to $0.364 \mathrm{eV}$, below the bottom of the surface state band $(\sim 0.16 \mathrm{eV})$.

these energies). The nondispersive character of these features and the linear troughs of the reconstruction pattern strongly suggest that the top layer behaves as a quasi-1D system. Indeed, were the electrons significantly confined within the reconstruction fringes [see Fig. 1(a)], they should behave as quasifree electrons along the [110] direction, whereas confinement should lead to a monotonous, nondispersive $\mathrm{BE}$ versus $k_{\|}$dependence along the perpendicular direction [110]. That a mild real-space corrugation, such as that of

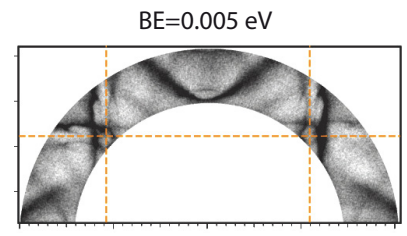

$\mathrm{BE}=0.133 \mathrm{eV}$
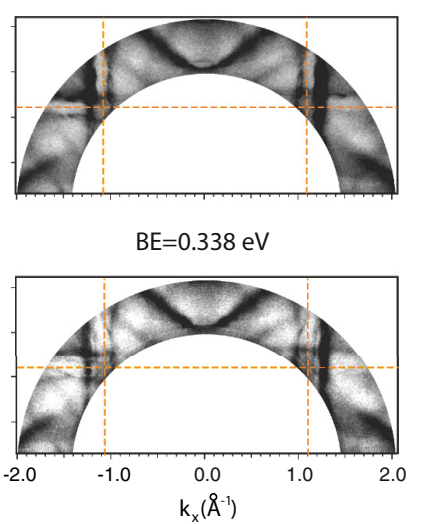
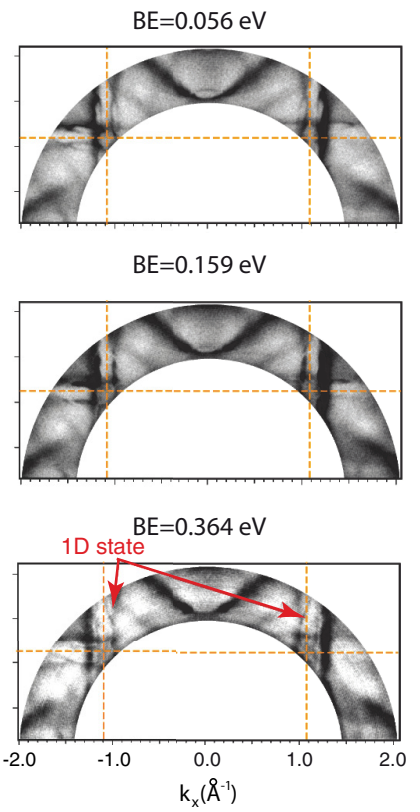

$\mathrm{BE}=0.159 \mathrm{eV}$

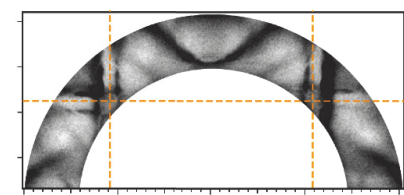

the $\mathrm{Au}(100)$ reconstruction fringes $(\approx 0.3 \AA)$, can confine electrons, is nothing strange: vicinal $\mathrm{Au}(788)$ surfaces exhibit similar confinement properties within its 3.8-nm terraces. ${ }^{25}$

A further insight into the behavior and nature of this electronic state never reported before is gained from the more detailed Fig. 4 equipotential surfaces depicted in Fig. 4. They were measured in a different experiment and on a different sample to discard possible artifacts, and cover a wider energy range to examine the behavior of the electronic state. Here,
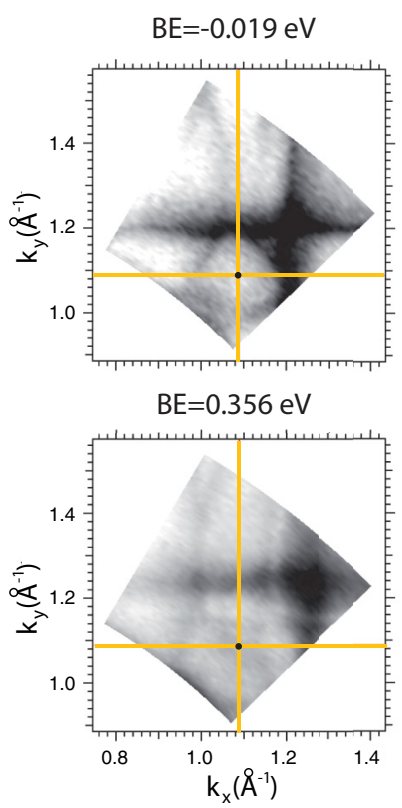

$\mathrm{BE}=0.056 \mathrm{eV}$

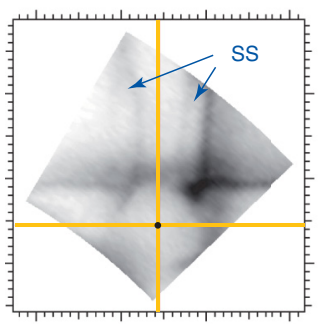

$\mathrm{BE}=0.431 \mathrm{eV}$

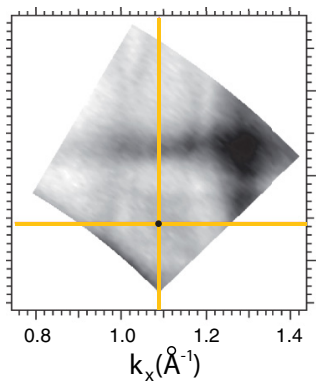

FIG. 4. (Color online) Constant energy maps in the region close to the $\bar{M}$ point for a large BE range, from $-0.019 \mathrm{eV}$ to $0.656 \mathrm{eV}$. Continuous yellow lines highlight the $(1 \times 1)$ Brillouin zone edges. For BE values smaller than $0.131 \mathrm{eV}$, oval shapes, characteristic of the surface state, can readily be seen. In the rest of the panels a number of lines parallel to [110] and [1110] high-symmetry directions (vertical and horizontal in the figure) are observed. In the constant energy map for $0.656 \mathrm{eV}$, dashed green lines show the shape expected for folding of the bulk $s p$ band in this reciprocal space region at the corresponding BE. The disagreement with the electronic states observed is remarkable. 
a succession of constant energy maps in the vicinity of the $\bar{M}$ point azimuthal angle $\phi \simeq 46^{\circ}$ measured from the [110] direction $\}$, with $\mathrm{BE}$ ranging from slightly above the Fermi level to well below the bottom of the Shockley surface state band, is shown. Arrows highlight the Shockley surface state (for 0.056-eV BE) and the 1-D electronic states (for 0.581-eV $\mathrm{BE}$ ). Again, there is a progressive shrinkage of the Shockley surface state oval on increasing the BE, readily seen in the first three panels, for $\mathrm{BE}<0.131 \mathrm{eV}$. Once this disappears, for $\mathrm{BE}$ about $0.16 \mathrm{eV}$, the remaining linear, nondispersive features mentioned above are again clearly seen. Since the BE lies below the bottom of the Shockley surface state band, the photoelectron intensity of these linear features must then come from a state other than the Shockley surface state. The sets of horizontal and vertical lines are surely contributions from perpendicular reconstruction domains. Both sets are better distinguished in Fig. 3 than in Fig. 4, probably due to the fact that different domains were present in more similar proportions in the sample of one experiment than in that of the other.

As mentioned above, a first idea about the origin of these electronic states is that they are due to backfolding of the bulk $s p$ band by the surface reconstruction, as observed at the Fermi energy in Fig. 2. A contour of the bulk $s p$ band calculated using a tight-binding code is shown in one of the panels of Fig. 4 (for 0.656-eV BE). The substantial disagreement allows us to discard a bulk origin for the electronic states. Now, from the more detailed and wider data set shown in Fig. 4, two revealing features can be observed: (a) there is a slight $k_{\|}$corrugation, and (b) the linear features disperse with BE. The corrugation is to be expected in case the reconstruction fringes are not totally decoupled from each other. This is a more realistic description than the electronic independence and total electronic confinement, ${ }^{25}$ which would call for a considerably high energy barrier between adjacent reconstruction fringes where the real-space corrugation is, as mentioned, of about $0.3 \AA$. This behavior has also been observed in other systems, ${ }^{26,27}$ recognized as quasi-1D.

An analysis of the energy dispersion of this hypothetical quasi-1D electronic state can shed light about its origin and nature. In Fig. 5(a) we plot the photoelectron intensity as a function of $k_{x}$ for several BEs. These curves have been obtained by making cuts at constant $k_{y}=1.25 \AA^{-1}$ in the equipotential surfaces of Fig. 4. In these curves, from four up to six maxima are observed. Cutting at different values of $k_{y}$ does not significantly alter the results due to the almost linear character of these states, save for the small corrugation mentioned before.

In Fig. 5(b), the intensity maxima from Fig. 5(a) have been plotted as a function of BE. The experimental points are grouped in four $X$-shaped "branches." Since the intensities are rather faint, branches are not always complete and may appear with only one of the two symmetrical slopes. One dashed, red line is a linear fit to the data of one branch, while the other dashed lines are guides to the view. The average slope of the linear fits is $7.5 \mathrm{eV} \AA^{-1}$. The distance between any one line and the nearest parallel one is $0.5 \times 0.168 \AA^{-1}=$ $0.084 \AA^{-1}=2 \pi / 26 a$. This is far from being a happy coincidence. It rather is a further support to the existence of a quasi-1D electronic state that, alike the bulk $3 \mathrm{D}$ and the surface $2 \mathrm{D}$ electronic states, becomes replicated with the (a)
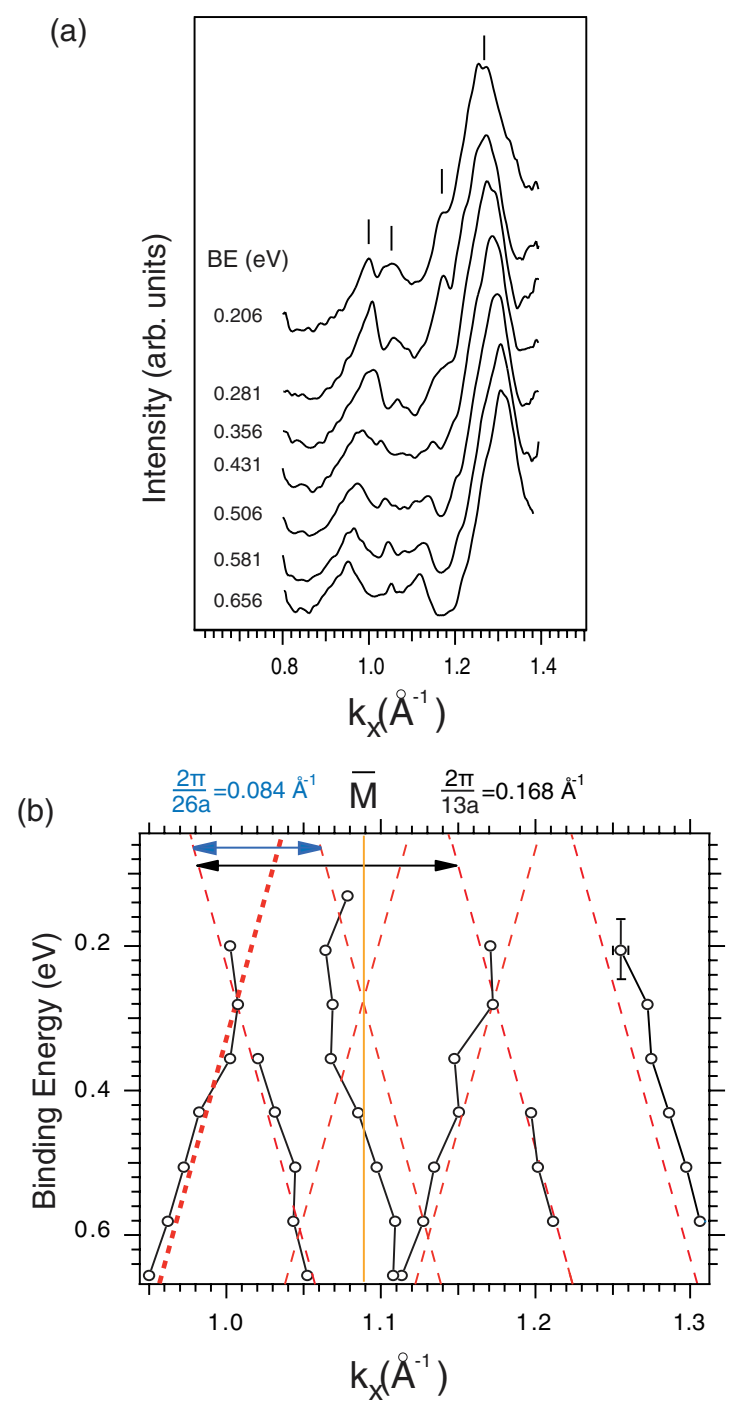

FIG. 5. (Color online) (a) Intensity cuts at constant $k_{y}=1.25 \AA^{-1}$ obtained from the data of Fig. 4 for different $\mathrm{BE}$ values below the bottom of the surface state. Tics mark the position of the intensity maxima in the top curve. (b) Plot of the intensity maxima from panel (a) against their corresponding BE. Red dots correspond to experimental points. The thick dashed line is a linear fit to the data. All other dashed lines are a guide to the eye and obtained by folding with the shown reciprocal lattice vector the thick dashed line. The estimated error bar for experimental points is shown for only one point for clarity. A vertical yellow (gray) line marks the edge of the first Brillouin zone.

topmost hexagonal layer periodicity. The fact that only the long period of the reconstruction [ $\times 26$ along [110] in Fig. 1(a)] enters in the replica scheme points also in the direction stated above: these quasi-1D states are linked to the 1D chainlike reconstruction fringes. And, more importantly, the presence of these quasi-1D states is an indication of significant lateral electronic confinement within the topmost hexagonal layer.

A scheme of the electronic energy in reciprocal space for such quasi-1D states is shown in Fig. 6. A constant energy map of this electronic structure would show a straight line in $k_{y}$ for a fixed $k_{x}$, which is precisely what is observed in Fig. 6(e). The vertical and horizontal straight lines seen in 
(a)

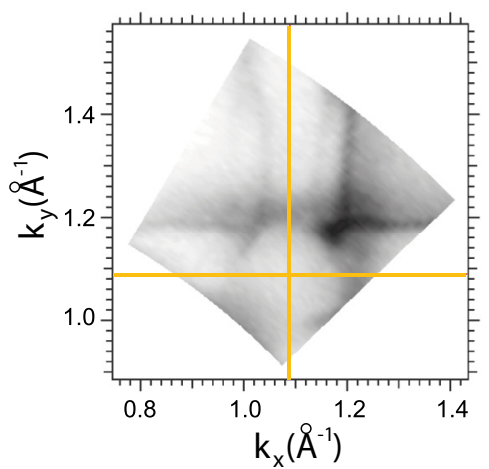

(e)

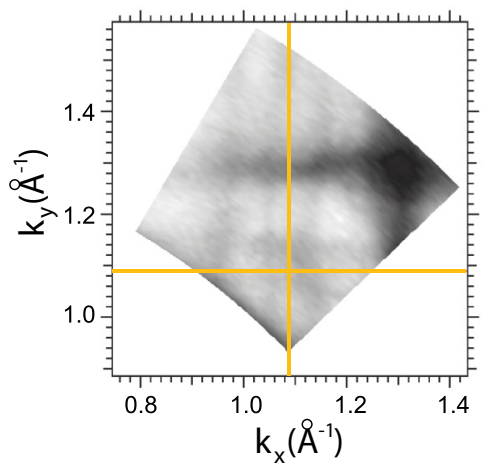

(b)

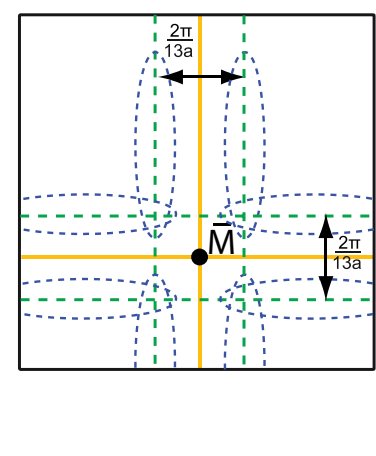

(f)

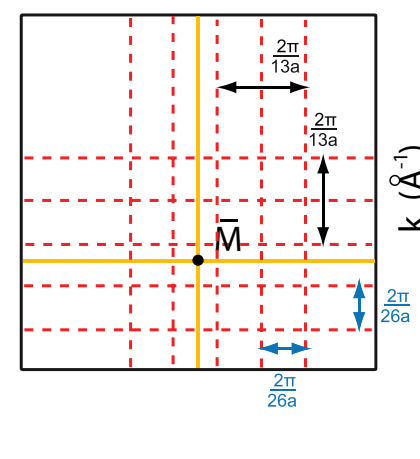

(c)

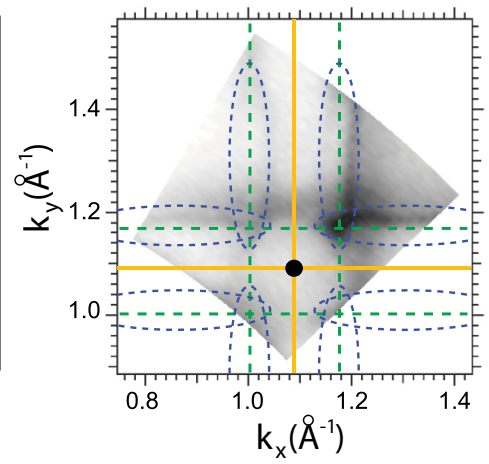

(g)

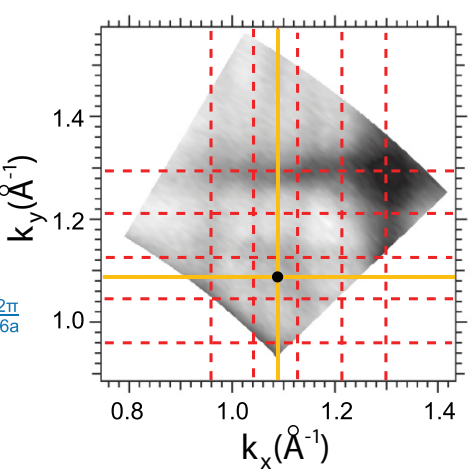

(d)

\section{Surface}

state

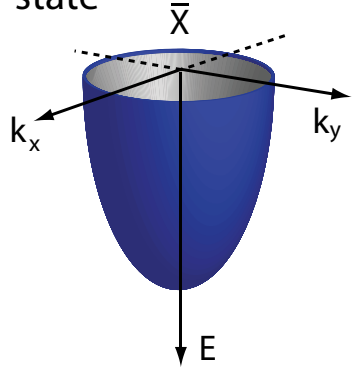

(h) 1-dim

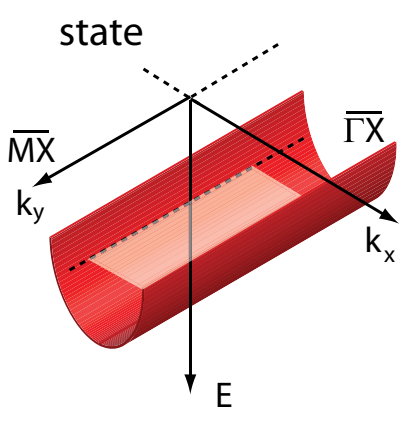

FIG. 6. (Color online) Two selected equipotential surfaces extracted from Fig. 4. Panels from (a) to (d) show the nature of the surface state while (e) to (h) explain the behavior of the 1D state. Panel (b) schematic surface state replicas (blue dash lines) while panel (f) is the same, but for the 1D state. These schemes are superimposed in (c) and (g), respectively. Panel (d) shows a schematic view of a 2D free-electron-like surface state in an energy vs parallel momentum representation. Panel (h) shows the same kind of representation for a $1 \mathrm{~S}$ surface state.

Fig. 6(e) suggest two orthogonal domains of this quasi-1D electronic state. Conversely, if we fix a constant $k_{y}$ value, cuts at different energies should exhibit a parabolic BE versus $k_{x}$ dispersion, as shown in Fig. 6(h). This is what is observed in Fig. 5(b). Dispersion is clearly seen, although we did not probe BEs high enough to get to the bottom of the band. The branches seen correspond to the 1D electronic state and its replicas, which can also be seen in Fig. 5(a). Not surprisingly, the replicas can be reproduced by shifting the 1D state along [110] by $2 \pi / 26 a$, which is the long reconstruction period.

\section{DISCUSSION}

We have shown that, in clean $\mathrm{Au}(100)$, both the $s p$ bulk band and the Shockley surface state become replicated with the periodicity of the reconstruction. The reason is simple: outgoing photoelectrons undergo an Umklapp scattering, absorbing an extra momentum that is an integer multiple of the reciprocal lattice reconstruction-induced superperiodicity. According to this, we expect processes that satisfy $\mathbf{k}_{\|, \mathbf{v a c}}=\mathbf{k}_{\|, \text {crystal }}+\mathbf{G}$, where $\mathbf{G}$ is a reciprocal lattice vector from the reconstructed surface. $\mathbf{G}$ can be either $n \times 2 \pi / 5 a=n \times 0.44 \AA^{-1}$ or $n \times$ $2 \pi / 26 a=n \times 0.084 \AA^{-1}, n$ being an integer, and $a=2.88 \AA$ the substrate surface square-cell size, as deduced from an analysis of the reconstructed unit cell. The fact that not all replicas are observed, and that their intensities are different, is related to matrix element effects (including photoelectron diffraction effects). ${ }^{28}$ As such, they change with the photon energy used, so that tuning the photon energy enhances the intensity of some replicas and weakens others. We worked at the photon energy $(41 \mathrm{eV})$ for which the intensity of the replica closest to the original Shockley surface state at $2 \pi / 13 a$ was higher.

That surface-related superstructures diffract outgoing photoelectrons, which absorb extra momentum from the upper layers and are emitted in different directions, is well known and has been reported in a number of papers. For instance, it has been observed in transition-metal surfaces with adsorbed species such as $\mathrm{Cl}$ on $\mathrm{Cu}(111)$ (Ref. 29) or $\mathrm{H}$ on W(100). ${ }^{30}$ In these heterogeneous systems, $d$ electrons undergo Umklapp processes involving reciprocal vectors of the adsorbate lattice. In clean surfaces, a similar behavior to what we report here has been observed in graphene nanostripes grown on stepped Ni(111) surfaces, ${ }^{31}$ terraces of stepped W(110) surfaces, ${ }^{32}$ and densely stepped $\mathrm{Au}(111)$ surfaces. ${ }^{33}$ In these, the step periodicity is responsible for the Umklapp process. Nevertheless, an Umklapp processes induced by surface reconstructions in homogeneous systems have never been reported before.

As for the quasi-1D electronic state, the first issue that arises is its origin. We have discarded that it is related both to the Shockley surface state and to a folding of the bulk $s p$ band. We assign these electronic states to the reconstructed topmost layer itself, corresponding mostly to the one hexagonal atomic layer at the surface of the reconstructed $\mathrm{Au}(100)$ crystal, corrugated 
to improve the registry with the (100) substrate. Thus, these states would correspond to the $s p$ electrons of the reconstructed layer. The ultimate driving-force for the reconstruction in $5 d$ metals is the compression that forces the topmost layer to adopt a denser hexagonal packing, a compression that is apparently originated by a transfer of $d$ electrons between the inner layers and the overlayer. The fine details and quantitative aspects of this charge transfer are still controversial. Fiorentini et $a l .{ }^{34}$ claimed that there is a $d$-charge depletion from the surface layer, whereas Annett and Inglesfield ${ }^{35}$ point towards an in-plane charge redistribution. Other authors have stressed that relativistic effects enhance the promotion of $d$ electrons into $s p$ states. According to recent theoretical calculations, ${ }^{21}$ enhanced $d-d$ hybridization is behind reconstruction in $5 d$ metals. If we analyze the structure of the reconstructed layer, it is clear that the fivefold periodicity is an efficient way to accommodate the hexagonal topmost layer periodicity in registry with the substrate. The hexagonal topmost layer becomes corrugated due to this accommodation, giving rise to the fivefold fringes along the [110] direction. A long-range periodicity is established also along the [110] direction, corresponding to the 26-fold superstructure. Unfortunately, due to the fact that other electronic states (Shockley surface state) are dominant in this energy range, no detailed information about the behavior of the 1D electronic states near the Fermi energy is available. However, a visual inspection of Fig. 5 reveals that the different branches of the 1D states are very close to each other near the Fermi energy, so close that they may cross and open an energy band gap. We can only speculate about that, but if this were the case, the 26 -fold periodicity would contribute to the energetic stability of the hexagonal topmost layer through a decrease of the electronic energy. This would suggest that the $1 \mathrm{D}$ electronic state could play an important role in promoting and stabilizing the reconstruction in $\mathrm{Au}(100)$.

That reconstruction and electron states are linked is proved by the fact that adsorption usually removes reconstruction. ${ }^{36-40}$ This is surely due to a charge transfer from the surface into the adsorbed species that reverses the mechanism, electronic in origin, that triggers reconstruction. On the other hand, there are experimental ${ }^{41}$ and theoretical ${ }^{42}$ evidences that point that $\mathrm{Au}(100)$ reconstructed areas have a "magic width," a multiple of five substrate atom rows (14.4 $\AA$ ), the width of the reconstruction fringes of Fig. 1(a). In Ref. 41, Au islands grown on reconstructed $\mathrm{Au}(100)$ tend to show widths of $30 \AA$ or less, and lengths range from 100 to $150 \AA$, figures that fit well with the reconstructed unit cell. Thus, five atom rows $\times 2.88 \AA=14.4 \AA$, so two reconstruction rows $\approx 30 \AA$. The long reconstruction period is $26 \times 2.88 \AA=$ $74.88 \AA$, so two long periods $\approx 150 \AA$, and one and a half long period $\approx 112 \AA$. In disordered $\mathrm{Au}(100)$ or $\operatorname{Pt}(100)$ surfaces, with crossed steps, sometimes the reconstruction is lifted, leaving a $(1 \times 1)$ area surrounded by monoatomic steps (see Figs. 11 and 12 of Ref. 15). This resembles a frustration mechanism due to the impossibility of developing a preferential reconstruction domain since reconstruction direction is preferentially determined by steps direction, as experimental data seem to suggest. ${ }^{15}$ From these experimental facts, it is tempting to argue that the 1D electronic state has its lowest energy for reconstruction fringes $14.4 \AA$ wide, and thus this "magic width" and its multiples lead to the maximum energy gain and the stabilization of the reconstruction. Detailed calculations would be needed to confirm this hypothesis.

\section{CONCLUSIONS}

We have studied the reconstructed $\mathrm{Au}(100)$ surface with ARPES, LEED, and STM. STM images show that the reconstruction can be described as a corrugated, compact hexagonal layer, with a periodicity of $(5 \times 26)$ in terms of the $(1 \times 1)$ bulk square lattice. In spite of this hexagonal topmost layer, the Shockley surface state persists below the reconstructed surface, an indication of symmetry decoupling between the electronic states of the bulk-terminated substrate and the overlayer. Both the bulk $s p$ band and the Shockley surface state replicate with the reconstruction periodicity as a consequence of Umklapp processes. We conclude that the measured electronic structure is fully consistent with the atomic structure of a corrugated topmost hexagonal layer on a bulk-terminated substrate.

We have observed a 1D electronic state, associated with the reconstruction fringes. The existence of such a state is a consequence of the strong lateral confinement within these fringes, $14.4 \AA$ wide, and of the relative decoupling of the reconstructed layer from the bulklike, underneath structure of square symmetry. This 1D state also replicates with the reconstruction periodicity $2 \pi / 26 a=0.084 \AA^{-1}$. We analyze the possible role of the $1 \mathrm{D}$ state in triggering and stabilizing the reconstruction, and in the uniform width of the reconstruction fringes and the fact that reconstruction patches mostly have an integer multiple of such fringes. To sum up, the rich electronic structure of reconstructed $\mathrm{Au}(100)$ contains three-dimensional, two-dimensional, and one-dimensional states and seems to be a suitable laboratory to test dimensionality effects and the interplay between surface and volume electronic structure.

\section{ACKNOWLEDGMENTS}

We acknowledge financial support from MINECO (Spain) under Grants No. MAT2010-21156-C03-02 and No. FIS201123230. The research leading to these results has received funding from the European Community's Seventh Framework Programme (FP7/2007-2013) under Grant Agreement No. 226716. 
${ }^{4}$ B. M. Ocko, D. Gibbs, K. G. Huang, D. M. Zehner, and S. G. J. Mochrie, Phys. Rev. B 44, 6429 (1991).

${ }^{5}$ D. Gibbs, B. M Ocko, D. M Zehner, and S. G. J. Mochrie, Phys. Rev. B 42, 7330 (1990).

${ }^{6}$ Y.-F. Liew and G.-C. Wang, Surf. Sci. 227, 190 (1990).

${ }^{7}$ K. Yamazaki, K. Takayanagi, Y. Tanishiro, and K. Yagi, Surf. Sci. 199, 595 (1988).

${ }^{8}$ P. Heimann, J. Hermanson, H. Miosga, and H. Neddermeyer, Phys. Rev. B 20, 3059 (1979).

${ }^{9}$ D. M. Kolb, W. Boeck, K.-M. Ho, and S. H. Liu, Phys. Rev. Lett. 47, 1921 (1981).

${ }^{10}$ G. Nicolay, F. Reinert, S. Hüfner, and P. Blaha, Phys. Rev. B 65, 033407 (2001).

${ }^{11}$ W. Chen, V. Madhavan, T. Jamneala, and M. F. Crommie, Phys. Rev. Lett. 80, 1469 (1998).

${ }^{12}$ F. Reinert and G. Nicolay, Appl. Phys. A 78, 817 (2004).

${ }^{13}$ A. Nuber, M. Higashiguchi, F. Forster, P. Blaha, K. Shimada, and F. Reinert, Phys. Rev. B 78, 195412 (2008).

${ }^{14}$ P. Heimann, J. Hermanson, H. Miosga, and H. Neddermeyer, Phys. Rev. Lett. 43, 1757 (1979).

${ }^{15}$ O. Rodriguez de la Fuente, M. A. González, and J. M. Rojo, Phys. Rev. B 63, 085420 (2001).

${ }^{16}$ R. H. M. Smit, C. Untiedt, A. I. Yanson, and J. M. van Ruitenbeek, Phys. Rev. Lett. 87, 266102 (2001).

${ }^{17}$ N. Nilius, M. V. Ganduglia-Pirovano, V. Brázdová, M. Kulawik, J. Sauer, and H. J. Freund, Phys. Rev. Lett. 100, 096802 (2008).

${ }^{18}$ E. Tosatti, S. Prestipino, S. Kostlmeier, A. D. Corso, and F. D. D. Tolla, Science 291, 288 (2001).

${ }^{19}$ N. Takeuchi, C. T. Chan, and K. M. Ho, Phys. Rev. B 43, 14363 (1991).

${ }^{20}$ G. Panaccione et al., Rev. Sci. Instrum. 80, 043105 (2009).

${ }^{21}$ P. Havu, V. Blum, V. Havu, P. Rinke, and M. Scheffler, Phys. Rev. B 82, 161418 (2010).

${ }^{22}$ V. Joco, Ph.D. thesis, Universidad Autoónoma, Madrid, Spain, 2008.

${ }^{23}$ S. Hüfner, Photoelectron Spectroscopy (Springer, Berlin, 1995).
${ }^{24}$ M. Lindroos and A. Bansil, Phys. Rev. Lett. 77, 2985 (1996).

${ }^{25}$ A. Mugarza, A. Mascaraque, V. Pérez-Dieste, V. Repain, S. Rousset, F. J. García de Abajo, and J. E. Ortega, Phys. Rev. Lett. 87, 107601 (2001).

${ }^{26}$ A. Mascaraque, L. Roca, J. Avila, S. Drouard, H. Guyot, and M. C. Asensio, Phys. Rev. B 66, 115104 (2002).

${ }^{27}$ H. W. Yeom, S. Takeda, E. Rotenberg, I. Matsuda, K. Horikoshi, J. Schaefer, C. M. Lee, S. D. Kevan, T. Ohta, T. Nagao, and S. Hasegawa, Phys. Rev. Lett. 82, 4898 (1999).

${ }^{28}$ E. L. Shirley, L. J. Terminello, A. Santoni, and F. J. Himpsel, Phys. Rev. B 51, 13614 (1995).

${ }^{29}$ D. Westphal and A. Goldmann, Surf. Sci. 126, 253 (1983).

${ }^{30}$ J. Anderson and G. Lapeyre, Phys. Rev. Lett. 36, 376 (1976).

${ }^{31}$ A. M. Shikin, S. A. Gorovikov, V. K. Adamchuk, W. Gudat, and O. Rader, Phys. Rev. Lett. 90, 256803 (2003).

${ }^{32}$ A. M. Shikin, A. Varykhalov, G. V. Prudnikova, V. K. Adamchuk, W. Gudat, and O. Rader, Phys. Rev. Lett. 93, 146802 (2004).

${ }^{33}$ A. Mugarza, A. Mascaraque, V. Repain, S. Rousset, K. N. Altmann, F. J. Himpsel, Y. M. Koroteev, E. V. Chulkov, F. J. Garcia de Abajo, and J. E. Ortega, Phys. Rev. B 66, 245419 (2002).

${ }^{34}$ V. Fiorentini, M. Methfessel, and M. Scheffler, Phys. Rev. Lett. 71, 1051 (1993).

${ }^{35}$ J. F. Annett and J. E. Inglesfield, J. Phys.: Condens. Matter 1, 3645 (1989).

${ }^{36}$ S. L. M. Schroeder, A. Neumann, T. Solomun, P. Lenz-Solomun, and K. Christmann, Surf. Sci. 337, 285 (1995).

${ }^{37}$ P. Gardner, M. Tshaus, R. Martin, and A. M. Bradshaw, Vacuum 41, 304 (1990).

${ }^{38}$ M. S. Zei and G. Ertl, Surf. Sci. 442, 19 (1999).

${ }^{39}$ P. van Beurden, B. S. Bunnik, G. J. Kramer, and A. Borg, Phys. Rev. Lett. 90, 066106 (2003).

${ }^{40}$ R. J. Behm, P. A. Thiel, P. R. Norton, and G. Ertl, J. Chem. Phys. 78, 7437 (1983).

${ }^{41}$ S. Günther, E. Kopatzki, M. C. Bartelt, J. W. Evans, and R. J. Behm, Phys. Rev. Lett. 73, 553 (1994).

${ }^{42}$ M. Nomura and X. Q. Wang, Phys. Rev. Lett. 81, 2739 (1998). 